\title{
ÁCIDO L-ASCÓRBICO: REAÇõES DE COMPLEXAÇÃo E DE ÓXIDO-REDUÇÃO COM ALGUNS ÍONS METÁLICOS DE TRANSIÇÃO
}

\author{
Adalgiza Fornaro e Nina Coichev \\ Instituto de Química da Universidade de São Paulo - CP 26077 - 05599-970 - São Paulo - SP
}

Recebido em 21/7/97; aceito em 17/12/97

\begin{abstract}
L-ASCORBIC ACID: COMPLEXATION AND REDOX REACTIONS WITH SOME TRANSITION METAL IONS. The strong reducing action of $L$-ascorbic acid (Vitamin $C$ ) are of fundamental interest in biochemical and related process. The oxidation of ascorbic acid by molecular oxygen and others oxidants are of fundamental importance, involving the intervention of transition metal ions as catalysts and the formation transition metal complexes of ascorbic acid as intermediates. The present article is intended to cover some aspects of the reactions of ascorbic acid and related compounds involving some transition metal ions.
\end{abstract}

Keywords: $L$-ascorbic acid; mechanism of the reaction; complexes of metal ions.

\section{INTRODUÇÃO}

O ácido $L$-ascórbico apresenta grande importância para sistemas bioquímicos, farmacológicos, eletroquímicos, processamento de alimentos e outros, sendo suas propriedades redox uma das características químicas de maior interesse ${ }^{1-3}$.

Dentro da abrangência dos aspectos da química e bioquímica do ácido ascórbico, no presente trabalho será dada atenção às reações de complexação e óxido-redução envolvendo alguns íons metálicos em meio aquoso. Não estão inclusos aspectos analíticos para a determinação do ácido ascórbico ou do ácido deidroascórbico. Vasta literatura encontra-se disponível, como por exemplo os artigos de revisão de Washko et alii ${ }^{4}$ e Pachla et alii $^{5}$, que dão ênfase à análise de ácido ascórbico em amostras biológicas, alimentos e produtos farmacêuticos.

$\mathrm{O}$ presente artigo abordará vários parâmetros cinéticos e termodinâmicos importantes para a compreensão da química do ácido ascórbico, contribuindo para o estudo de reatividade, equilíbrios químicos, bioinorgânica e também para o desenvolvimento de métodos analíticos. Na apreciação da literatura abordada a seguir, optou-se por apresentar as sequências de reações conforme consta no artigo original.

\section{EQUILÍBRIOS ÁCIDO-BASE E DE ÓXIDO- REDUÇÃO DO ÁCIDO $L$-ASCÓRBICO}

Vitamina $\mathrm{C}$ é o termo frequentemente usado para referir-se ao ácido $L$-ascórbico, figura 1 (I), e ao seu produto de oxidação inicial, o ácido $L$-deidroascórbico, sendo que ambos apresentam atividade como vitaminas. No entanto, os isomêros (II), (III) e (IV) (Fig. 1) praticamente não apresentam atividades vitamínicas $^{1-3}$.

$\mathrm{O}$ ácido $L$-ascórbico $\left(\mathrm{H}_{2} \mathrm{~A}\right)$ possui a estrutura de um diol que é oxidado formando o ácido $L$-deidroascórbico $\left(\mathrm{A}_{\mathrm{ox}}\right)$, equação

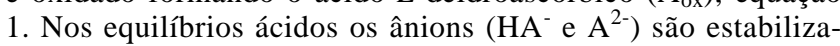
dos pela distribuição da carga através do sistema enona $\mathrm{O} 1=\mathrm{C} 1$ $\mathrm{C} 2=\mathrm{C} 3-\mathrm{O} 3$, sendo o hidrogênio mais ácido o do grupo hidroxil ligado ao $\mathrm{C} 3^{1-3}$.

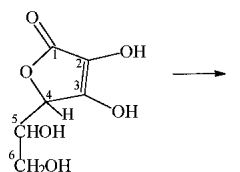

$\mathrm{H}_{2} \mathrm{~A}$

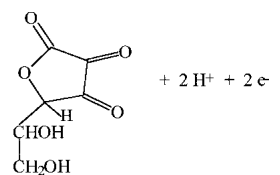

$\mathrm{A}_{0 \mathrm{x}}$

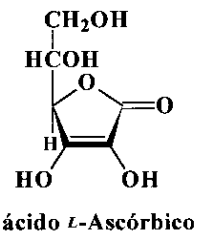

I

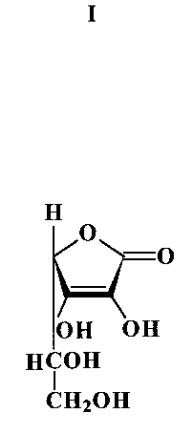

ácido $\mathrm{D}$-Ascórbico

II

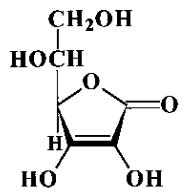

ácido $D$-Isoascórbico

III
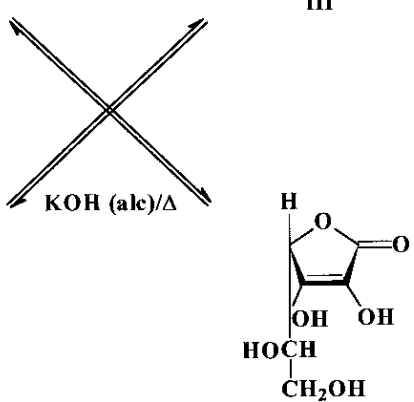

ácido $L$-Isoascórbico

IV
Figura 1. Estereoisômeros do ácido ascórbico em meio alcoólico e de KOH a quente ${ }^{3}$.

Os processos redox do ácido ascórbico, representados pelas equações (2) a (4), são reversíveis com a formação de radicais livres intermediários. A perda de um elétron (eq. 2), leva à formação do radical $L$-ascorbato intermediário, $\mathrm{HA}^{\Uparrow}$, também chamado ácido monodeidroascórbico ou ácido semideidroascórbico. A espécie $\mathrm{HA}^{\Uparrow}$ se dissocia $\left(\mathrm{pK}_{\mathrm{a}}=-0,45\right)$ formando o radical $A^{-\Uparrow}$ (eq. 3). A perda do segundo elétron leva à formação do ácido deidroascórbico (eq. 4) (-8 $^{\text {. }}$.

É importante observar que para a oxidação do ácido ascórbico no total dois prótons e dois elétrons são perdidos. No esquema 1 estão apresentados os valores das constantes ácidas e os potenciais de redução para as espécies do ácido ascórbico em solução aquosa.

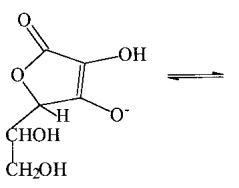

$\mathrm{HA}^{-}$

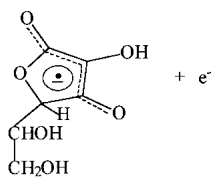

$\mathrm{HA}^{*}$ 


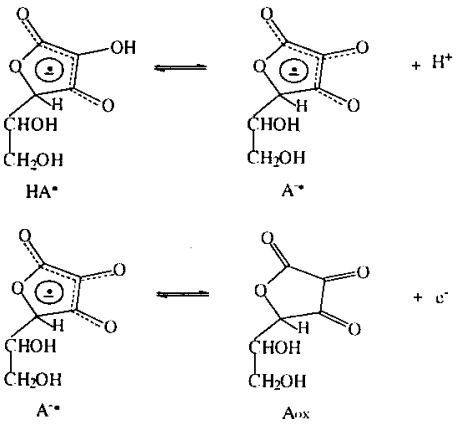

No esquema 1 observa-se que os potenciais padrão de redução diminuem na sequência $\mathrm{E}^{\mathrm{o}}\left(\mathrm{A}_{\mathrm{ox}} / \mathrm{H}_{2} \mathrm{~A}\right)>\mathrm{E}^{\mathrm{o}}\left(\mathrm{A}_{\mathrm{ox}} / \mathrm{HA}^{-}\right)>$ $\mathrm{E}_{\mathrm{o}}\left(\mathrm{A}_{\mathrm{ox}} / \mathrm{A}^{2-}\right)$. Assim, as propriedades redutoras dessas espécies seguem a seguinte ordem $\mathrm{A}^{2-}>\mathrm{HA}^{-}>\mathrm{H}_{2} \mathrm{~A}^{6,9}$.

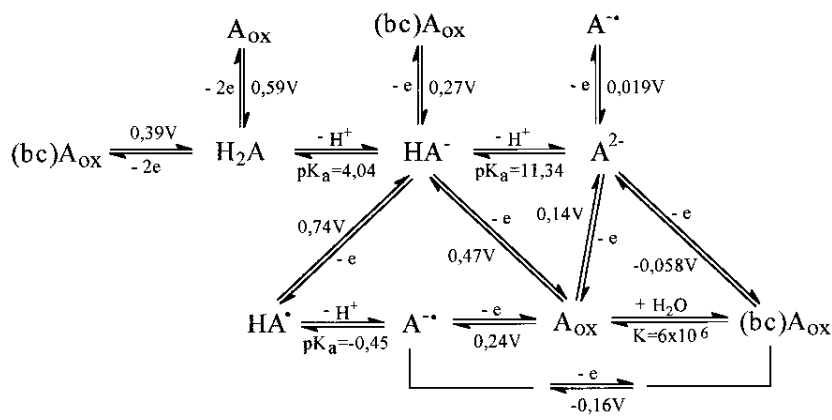

Esquema 1. Potenciais padrão de redução vs EPH (Eletrodo Padrão de Hidrogênio) e constantes de acidez do ácido L-ascórbico e espécies relacionadas, a $25^{\circ} \mathrm{C}$ e força iônica $0,2 \mathrm{~mol} \mathrm{~L}^{-1}$ 6, 9 .

Em meio aquoso é importante considerar que o ácido $L$ deidroascórbico predomina $(99 \%)$ na forma da espécie bicíclica hidratada, (bc) $\mathrm{A}_{\mathrm{ox}}$, equação (5), apresentando uma constante de equilíbrio elevada ${ }^{1,6}, \mathrm{~K}=6 \times 10^{6} \mathrm{~mol} \mathrm{~L}^{-1}$.

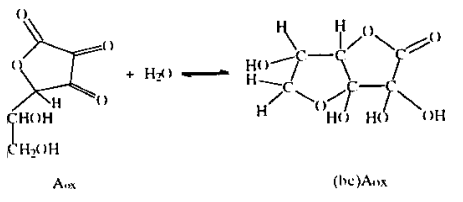

A reação de decomposição do ácido deidroascórbico (eq.6) ocorre através da abertura do anel por hidrólise com a formação do ácido 2,3-diceto- $L$-gulônico que é considerada irreversível ${ }^{4}$.

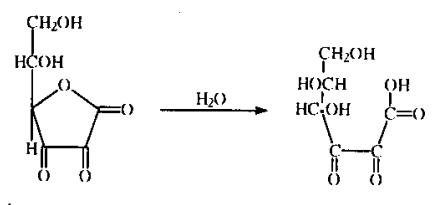

\section{REAÇÕES DE COMPLEXAÇÃO DO ÁCIDO ASCÓRBICO COM ALGUNS ÍONS METÁLICOS DE TRANSIÇÃO}

O ácido ascórbico forma complexos com íons metálicos do tipo MA e MHA, dependendo do $\mathrm{pH}$ do meio, mas esses complexos são relativamente instáveis ${ }^{10,11}$. Na tabela 1 estão apresentados valores de constantes de equilíbrio entre o ácido ascórbico e alguns íons metálicos, determinados como espécies intermediárias nos mecanismos de reação de oxidação do ácido ascórbico por íons metálicos de transição. Observa-se que a maioria desses complexos são do tipo 1:1 com a espécie $\mathrm{HA}^{-} \mathrm{e}$ apresentam baixos valores de constante de equilíbrio. Enquanto que o complexo de íon ouro é do tipo 1:2 e o de íon prata é formado com a espécie $\mathrm{A}^{2-}$ e apresentam valores de constante de equilíbrio mais altos ${ }^{1,2}$.

A combinação de problemas associados às reações de óxido redução rápidas, hidrólise e precipitação de íons metálicos faz com que sejam poucos os trabalhos sobre a formação de complexos com o íon ascorbato $\left(\mathrm{A}^{2-}\right)$ em meio de baixa acidez, isto é, em condições onde complexos quelatos desprotonados devem ser formados ${ }^{2}$.

Não existem muitos trabalhos sobre o isolamento de complexos sólidos de ácido ascórbico. Alguns complexos cristalinos descritos para uma variedade de íons metálicos de transição como $\mathrm{TiO}^{2+}, \mathrm{Cr}^{3+}, \mathrm{Mn}^{2+}, \mathrm{Co}^{2+}, \mathrm{Ni}^{2+}$ e $\mathrm{Zn}^{2+}$, foram preparados e isolados a partir da reação do sulfato do íon metálico com ácido ascórbico na presença de hidróxido de bário em ausência total de oxigênio ${ }^{12,13}$.

Martinez e Uribe ${ }^{14}$ isolaram um sólido azul intenso a partir da mistura do íon ferro(III) com ascorbato, ao qual foi dada a fórmula $\left[\mathrm{Fe}(\mathrm{HA})_{2}\right]^{+}$. O espectro de absorção do complexo é similar ao do intermediário azul observado em estudos em meio aquoso da oxidação do ácido ascórbico por íons ferro(III). Os autores concluíram a partir de estudos químicos e de espectros Mössbauer que o íon ferro está presente no estado de oxidação (III). A partir de espectros RMN eles também sugeriram que o ácido ascórbico está ligado como um quelato bidentado ao ferro através dos átomos de oxigênio dos carbonos (2) e (3), e a estrutura sugerida pelos autores está apresentada na figura $2^{14}$.

Uma série de complexos de ácido ascórbico do tipo cis[Pt $\left(\mathrm{RNH}_{2}\right)$ (ascorbato)] foi sintetizada e isolada, sendo o ligante, $\mathrm{R}$, a espécie cis-1,2-diaminocicloexano (cis-dach). A estrutura determinada por cristalografia de Raios-X está apresentada na figura 3. Como esperado, o ascorbato é um ligante bidentado, e no

Tabela 1. Valores de constantes de equilíbrio para complexos de ácido ascórbico com alguns íons metálicos de transição a $25^{\circ} \mathrm{C}$ em meios de diferente força iônica (I) ${ }^{1}$.

\begin{tabular}{cccc}
\hline Íon metálico & Equilíbrio & $\log \mathrm{K}$ & $\mathrm{I}\left(\mathrm{mol} \mathrm{L}^{-1}\right)$ \\
\hline $\mathrm{Mn}^{2+}$ & {$[\mathrm{MnHA}]^{+} /\left[\mathrm{Mn}^{2+}\right] \cdot\left[\mathrm{HA}^{-}\right]$} & 1,1 & 0 \\
$\mathrm{Fe}^{2+}$ & {$[\mathrm{FeHA}]^{+} /\left[\mathrm{Fe}^{2+}\right] \cdot\left[\mathrm{HA}^{-}\right]$} & 0,21 & 1,0 \\
& {$[\mathrm{FeA}] /\left[\mathrm{Fe}^{2+}\right] \cdot\left[\mathrm{A}^{2-}\right]$} & 1,99 & 1,0 \\
$\mathrm{Co}^{2+}$ & {$[\mathrm{CoHA}]^{+} /\left[\mathrm{Co}^{2+}\right] \cdot\left[\mathrm{HA}^{-}\right]$} & 1,4 & 0 \\
$\mathrm{Ni}^{2+}$ & {$[\mathrm{NiHA}]^{+} /\left[\mathrm{Ni}^{2+}\right] \cdot\left[\mathrm{HA}^{-}\right]$} & 1,57 & 0 \\
$\mathrm{Cu}^{2+}$ & {$[\mathrm{CuHA}]^{+} /\left[\mathrm{Cu}^{2+}\right] \cdot\left[\mathrm{HA}^{-}\right]$} & 4,7 & 0,1 \\
$\mathrm{Cu}^{2+}$ & {$\left[\mathrm{Cu}\left(\mathrm{H}_{2} \mathrm{~A}\right)\right]^{2+} /\left[\mathrm{Cu}^{2+}\right] \cdot\left[\mathrm{HA}^{-}\right] \cdot\left[\mathrm{H}^{+}\right]$} & 3,66 & 0,1 \\
$\mathrm{Ag}^{+}$ & {$[\mathrm{AgA}]^{-} /\left[\mathrm{Ag}^{+}\right] \cdot\left[\mathrm{A}^{2-}\right]$} & 1,89 & 0,1 \\
$\mathrm{Au}^{3+}$ & {$[\mathrm{AuHA}]^{2+} /\left[\mathrm{Au}^{3+}\right] \cdot\left[\mathrm{HA}^{-}\right]$} & 3,55 & 0,1 \\
& {$\left[\mathrm{Au}(\mathrm{HA})_{2}\right]^{+} /\left[\mathrm{Au}^{3+}\right] \cdot\left[\mathrm{HA}^{-}\right]^{2}$} & 0,1 \\
\hline
\end{tabular}


<smiles></smiles>

Figura 2. Estrutura sugerida para o complexo de Ferro(III)/ácido ascórbico $^{14}$.

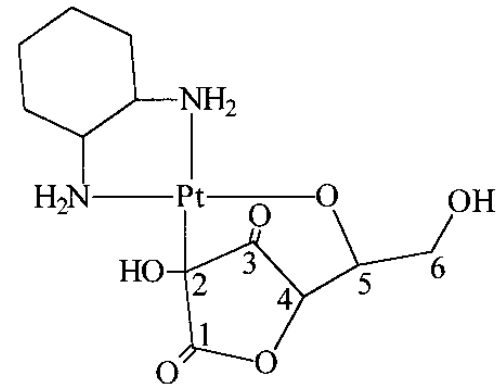

Figura 3. Estrutura do complexo cis-1,2-diaminociclohexanoplatina(II)/ácido ascórbico ${ }^{15}$.

caso da Pt a ligação não ocorre pelos oxigênios (2) e (3), mas sim por meio do carbono (2) e do oxigênio ligado ao carbono $(5)^{15}$

Apesar das muitas especulações sobre as estruturas de complexos envolvendo o ácido ascórbico, a estrutura do tipo da figura 3, tem sido considerada apenas para complexos com platina ${ }^{2}$.
Dados da literatura têm sugerido que para complexos formados com uma variedade de cátions divalentes como $\mathrm{Ca}^{2+}, \mathrm{VO}^{2+}$, $\mathrm{Mn}^{2+}, \mathrm{Zn}^{2+}$ e $\mathrm{Cd}^{2+}$, o ácido ascórbico atua como ligante monodentado. Estudos de $\mathrm{RMN}$ de ${ }^{13} \mathrm{C}$ levaram à conclusão de que para os íons metálicos $\mathrm{Co}^{2+}, \mathrm{Fe}^{2+}$ e $\mathrm{Mn}^{2+}$, em pH 8,5, os complexos ascorbato-íon metálico são formados por quelação com $\mathrm{O}(2)$ e $\mathrm{O}(3)$ do íon ascorbato ${ }^{16}$. Estudos similares com $\mathrm{Ni}^{2+}$ em presença de ácido ascórbico mostraram que o ascorbato também está ligado ao íon metalíco pelos oxigênios (2) e (3) ${ }^{17}$.

Poucos trabalhos são encontrados na literatura sobre a determinação das estruturas de complexos estáveis em solução entre ascorbato e íons metálicos. No entanto, muitos estudos comprovam a formação de complexos como espécies intermediárias durante as reações de oxidação do ácido ascórbico por íons metálicos ${ }^{2}$.

\section{REAÇÕES DE OXIDAÇÃO DO ÁCIDO ASCÓRBICO POR COMPLEXOS DE ÍONS METÁLICOS DE TRANSIÇÃO}

Uma série de estudos de reações de oxidação do ácido $L$ ascórbico foi realizada empregando-se complexos de diversos íons metálicos, e uma valiosa revisão sobre o assunto foi publicada por Davies ${ }^{2}$.

É importante lembrar que as reações de transferência de prótons que ocorrem simultaneamente com as reações de óxidoredução aumentam a complexidade desses estudos ${ }^{18}$.

Para as reações envolvendo a oxidação do ácido ascórbico, a maioria dos autores tem proposto a formação do radical ascorbato, protonado $\left(\mathrm{HA}^{\Uparrow}\right)$ ou não $\left(\mathrm{A}^{-\Uparrow}\right)$, na etapa determinante da reação ${ }^{19-23}$

A tabela 2 apresenta os valores da constante de velocidade de reação a $25^{\circ} \mathrm{C}$, para a oxidação do ácido $L$-ascórbico por

Tabela 2. Alguns exemplos de trabalhos realizados sobre a determinação da constante de velocidade de reação de oxidação do ácido $L$-ascórbico, a $25^{\circ} \mathrm{C}$.

\begin{tabular}{|c|c|c|}
\hline Pares redox & $\mathrm{k}\left(\mathrm{mol}^{-1} \mathrm{~L} \mathrm{~s}^{-1}\right)$ & Referências \\
\hline $\mathrm{Cu}^{2+} / \mathrm{H}_{2} \mathrm{~A}$ & $5,8 \times 10^{-1}$ & 24 \\
\hline$[\mathrm{CuCl}]^{+} / \mathrm{HA}^{-}$ & $2,8 \times 10^{2}$ & 24 \\
\hline $\mathrm{Cu}(\mathrm{Ac})^{+} / \mathrm{HA}^{-}$ & $3,1 \times 10^{4}$ & 24 \\
\hline $\mathrm{Co}^{3+} / \mathrm{H}_{2} \mathrm{~A}$ & $2,8 \times 10^{2}$ & 8 \\
\hline$[\mathrm{CoOH}]^{2+} / \mathrm{H}_{2} \mathrm{~A}$ & $7,3 \times 10^{5}$ & 8 \\
\hline$\left[\mathrm{Co}(\text { phen })_{3}\right]^{3+} / \mathrm{HA}^{-}$ & $4,0 \times 10^{-1}$ & 25 \\
\hline$\left[\mathrm{Co}(\text { phen })_{3}\right]^{3+} / \mathrm{A}^{2-}$ & $5,8 \times 10^{6}$ & 25 \\
\hline$\left[\mathrm{Co}(\text { bipy })_{3}\right]^{3+} / \mathrm{HA}^{-}$ & $1,3 \times 10^{-1}$ & 25 \\
\hline$\left[\mathrm{Co}(\text { bipy })_{3}\right]^{3+} / \mathrm{A}^{2-}$ & $2,1 \times 10^{6}$ & 25 \\
\hline$\left[\mathrm{Co}\left(\mathrm{NH}_{3}\right)_{6}\right]^{3+} / \mathrm{HA}^{-}$ & 2,7 & 26 \\
\hline$\left[\mathrm{Co}\left(\mathrm{NH}_{3}\right)_{5} \mathrm{OH}\right]^{2+} / \mathrm{HA}^{-}$ & $1,9 \times 10^{-1}$ & 27 \\
\hline$\left[\mathrm{Co}\left(\mathrm{NH}_{3}\right)_{5} \mathrm{OH}\right]^{2+} / \mathrm{A}^{2-}$ & $2,7 \times 10^{2}$ & 27 \\
\hline$\left[\mathrm{Co}\left(\mathrm{C}_{2} \mathrm{O}_{4}\right)_{3}\right]^{3-} / \mathrm{H}_{2} \mathrm{~A}$ & $1,2 \times 10^{-4}$ & 28 \\
\hline$\left[\mathrm{Co}\left(\mathrm{C}_{2} \mathrm{O}_{4}\right)_{3}\right]^{3-} / \mathrm{HA}^{-}$ & $4,1 \times 10^{-3}$ & 28 \\
\hline$\left[\mathrm{Co}\left(\mathrm{C}_{2} \mathrm{O}_{4}\right)_{3}\right]^{3-} / \mathrm{A}^{2-}$ & $2,0 \times 10^{1}$ & 23 \\
\hline$\left[\mathrm{Co}\left(\mathrm{C}_{2} \mathrm{O}_{4}\right)_{3}\right]^{3-} / \mathrm{HA}^{-}$ & $7,0 \times 10^{-3}$ & 29,30 \\
\hline$\left[\mathrm{Co}\left(\mathrm{C}_{2} \mathrm{O}_{4}\right)_{3}\right]^{3-} / \mathrm{A}^{2-}$ & $1,1 \times 10^{1}$ & 29,30 \\
\hline$\left[\mathrm{Fe}\left(\mathrm{H}_{2} \mathrm{O}\right)_{6}\right]^{3+} / \mathrm{H}_{2} \mathrm{~A}$ & 3,4 & 19 \\
\hline$\left[\mathrm{Fe}\left(\mathrm{H}_{2} \mathrm{O}\right)_{5} \mathrm{OH}\right]^{2+} / \mathrm{H}_{2} \mathrm{~A}$ & $1,7 \times 10^{3}$ & 19 \\
\hline$\left[\mathrm{Fe}(\mathrm{CN})_{6}\right]^{3-} / \mathrm{H}_{2} \mathrm{~A}$ & $5,7 \times 10^{-1}$ & 20 \\
\hline$\left[\mathrm{Fe}(\mathrm{CN})_{6}\right]^{3-} / \mathrm{HA}^{-}$ & $8,6 \times 10^{3}$ & 20 \\
\hline$\left[\mathrm{Fe}\left(\mathrm{C}_{2} \mathrm{O}_{4}\right)_{3}\right]^{3-} / \mathrm{HA}^{-}$ & $2,1 \times 10^{4}$ & 21 \\
\hline$\left[\mathrm{Fe}(\mathrm{CN})_{6}\right]^{3-} / \mathrm{A}^{2-}$ & $2 \times 10^{7}$ & 23 \\
\hline$\left[\mathrm{Fe}(\text { phen })_{3}\right]^{3+} / \mathrm{H}_{2} \mathrm{~A}$ & $2 \times 10^{5}$ & 28 \\
\hline$\left[\mathrm{Fe}(\text { phen })_{3}\right]^{3+} / \mathrm{HA}^{-}$ & $6 \times 10^{8}$ & 28 \\
\hline$[\mathrm{Fe}(\mathrm{III}) / \mathrm{EDTA}] / \mathrm{HA}^{-}$ & $2,8 \times 10^{3}$ & 31 \\
\hline$\left[\mathrm{Fe}(\text { bipy })_{3}\right]^{3+} / \mathrm{HA}^{-}$ & $5,4 \times 10^{8}$ & 32 \\
\hline$\left[\mathrm{Fe}(\text { bipy })_{2}\right]^{3+} / \mathrm{H}_{2} \mathrm{~A}$ & $8,2 \times 10^{-2}$ & 33 \\
\hline $\mathrm{Mn}^{3+} / \mathrm{H}_{2} \mathrm{~A}$ & $7 \times 10^{3}$ & 32 \\
\hline$[\mathrm{MnOH}]^{2+} / \mathrm{H}_{2} \mathrm{~A}$ & $8 \times 10^{4}$ & 32 \\
\hline
\end{tabular}


alguns íons complexos de cobalto(III), ferro(III), cobre(II) e manganês(III). Outros estudos envolvendo complexos com outros íons metálicos de transição estão resumidos no artigo de revisão de Davies ${ }^{2}$. Artigos mais recentes envolvem diferentes complexos de $\mathrm{Mn}(\mathrm{III})^{34,35}, \mathrm{Ni}(\mathrm{IV})^{36}$ e Ru(III) ${ }^{37}$.

A partir dos exemplos apresentados na tabela 2 pode-se observar a importância do $\mathrm{pH}$, do íon metálico e da presença de diferentes ligantes complexados a esses íons metálicos para a velocidade de reação de oxidação do ácido ascórbico.

Por exemplo, o efeito do $\mathrm{pH}$ pode ser observado comparando-se a velocidade de reação para o $\left[\mathrm{Co}(\text { phen })_{3}\right]^{3-}$, que em meio básico, região em que predomina a espécie $\mathrm{A}^{2-}$, é $1,4 \times 10^{7}$ vezes maior que em meio de diferente acidez, onde predomina a espécie $\mathrm{HA}^{-}$.

O efeito da presença de ligantes na velocidade da reação pode ser observado para o íon Fe(III), em meio de igual acidez (HA ${ }^{-}$ ), a velocidade da reação é $2,1 \times 10^{5}$ vezes maior quando esse íon metálico está complexado com fenantrolina comparativamente à complexação com EDTA.

Também verifica-se, a partir da Tabela 2, que o íon ferro(III) reage muito mais rapidamente com o ácido ascórbico que o cobalto (III); por exemplo, o $\left[\mathrm{Fe}\left(\mathrm{C}_{2} \mathrm{O}_{4}\right)_{3}\right]^{3-}$ reage $5,1 \times 10^{6}$ vezes mais rapidamente com o ácido ascórbico do que o $\left[\mathrm{Co}\left(\mathrm{C}_{2} \mathrm{O}_{4}\right)_{3}\right]^{3-}$ em meio no qual predomina a espécie $\mathrm{HA}^{-}$.

\subsection{Reações de oxidação do ácido ascórbico por complexos de $\mathrm{Fe}($ III)}

O ácido ascórbico apresenta várias funções importantes no corpo, estando envolvido no metabolismo de vários tecidos e em múltiplos processos bioquímicos.

Alguns estudos concluem que a absorção de íons ferro, especialmente quando complexado com glicina, é favorecida em presença de ácido ascórbico devido a reação de óxido-redução, que mantém a forma biodisponível, íons Fe(II), em solução. Sendo a acidez do meio muito importante na cinética dessa reação, assim como na solubilidade de sais de íons Fe(II)/(III) ${ }^{38-41}$.

$\mathrm{O}$ mecanismo originalmente proposto para a reação do íon $\mathrm{Fe}(\mathrm{III})$ aquoso com $\mathrm{H}_{2} \mathrm{~A}$ é de um processo de transferência de elétrons por esfera interna que envolve a formação de um complexo quelato com $\mathrm{Fe}(\mathrm{III})$ (eq. 7 e 8). Em seguida, um elétron é transferido a partir do ascorbato para o $\mathrm{Fe}(\mathrm{III})$ para produzir o radical de complexo-Fe(III) (eq. 9). Este, então, é convertido para o ácido deidroascórbico $\left(\mathrm{A}_{\mathrm{ox}}\right)$ (eq. 9 a 11). A etapa determinante deste mecanismo é a formação do complexo intermediário, $\mathrm{FeHA}^{2+42-44}$

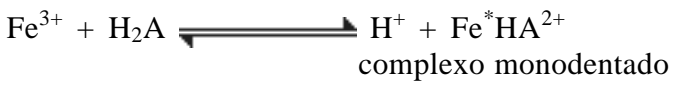

$$
\mathrm{Fe}^{3+} \mathrm{HA}^{2+} \rightleftharpoons \underset{\text { complexo quelato }}{\underset{\text { FeHA }}{2+}} \mathrm{K}=0,55 \mathrm{~mol} \mathrm{~L}^{-1}
$$

Foi proposto que no rearranjo do complexo monodentado para quelato (eq. 7 e 8), ocorre uma transferência intramolecular de elétrons a partir do ascorbato para o íon férrico. O complexo do metal reduzido dissocia-se para produzir $\mathrm{Fe}^{2+}$ e um radical ascorbato (eq. 10) que sofre reações rápidas com outro íon $\mathrm{Fe}^{3+}$ para produzir mais um íon $\mathrm{Fe}^{2+}$ e o ácido deidroascórbico $\left(\mathrm{A}_{\mathrm{ox}}\right)^{44}$ :

$$
\begin{aligned}
& \mathrm{FeHA}^{2+} \rightleftharpoons\left(\mathrm{Fe}^{2+} \ldots \mathrm{HA}^{\bullet}\right) \\
& \left(\mathrm{Fe}^{2+} \ldots \mathrm{HA}^{\bullet} \rightleftharpoons \mathrm{Fe}^{2+}+\mathrm{H}^{+}+\mathrm{A}^{-}\right. \\
& \mathrm{A}^{-\bullet}+\mathrm{Fe}^{3+} \rightarrow \mathrm{Fe}^{2+}+\mathrm{A}_{\mathrm{ix}}
\end{aligned}
$$

Em vários estudos a formação do complexo $\mathrm{Fe}(\mathrm{III})$-ascorbato, como uma espécie azul com máximo de absorção em $\lambda=550 \mathrm{~nm}$ $\left(\varepsilon=11 \mathrm{~L} \mathrm{~mol}^{-1} \mathrm{~cm}^{-1}\right)^{19,31,42-44}$, foi detectada como um intermediário, durante a redução de $\mathrm{Fe}(\mathrm{III})$ a $\mathrm{Fe}(\mathrm{II})$ em meio aquoso.
Lawrence e Ellis ${ }^{44}$ examinaram essa reação através de medições espectrofotométricas com variações de espectro entre 450$650 \mathrm{~nm}$ e detectaram um rápido aumento inicial na absorbância ( $\lambda$ $=550 \mathrm{~nm}$ ), atribuído à formação de um complexo instável, seguido de uma lenta diminuição dessa absorbância. A esse complexo intermediário foi atribuída a fórmula $[\mathrm{FeHA}]^{2+}$. Esses autores determinaram uma constante de equilíbrio para o complexo intermediário ao redor de $0,55 \mathrm{~mol} \mathrm{~L}^{-1}$ (eq. 8) (4) $^{4}$.

Um outro estudo cuidadoso em condições de excesso de íons $\mathrm{Fe}(\mathrm{III})$, reagindo com ácido ascórbico, mostrou que a reação ocorre em dois estágios, primeiro com um aumento rápido da absorbância, em $560 \mathrm{~nm}\left(\mathrm{t}_{1 / 2}=10-20 \mathrm{~ms}\right)$ seguido de uma diminuição lenta $\left(\mathrm{t}_{1 / 2}=200-400 \mathrm{~ms}\right)$. A etapa rápida desta reação foi atribuída à substituição de moléculas de $\mathrm{H}_{2} \mathrm{O}$ pela molécula de $\mathrm{H}_{2} \mathrm{~A}$ com formação das espécies [ $\left.\mathrm{Fe}(\mathrm{HA})\right]^{2+}$ (eq. 14 e 15$)$ e $\left[\mathrm{Fe}\left(\mathrm{H}_{2} \mathrm{~A}\right)\right]^{3+}$ (eq. 16). Para simplificação os autores desses estudos omitiram o número de moléculas de água coordenadas aos complexos $[\mathrm{Fe}(\mathrm{HA})]^{2+}$ e $\left[\mathrm{Fe}\left(\mathrm{H}_{2} \mathrm{~A}\right)\right]^{3+}$ (eq. 14-17), embora alguns trabalhos ${ }^{42-44}$ apresentem esses complexos com $\mathrm{O}$ ácido ascórbico agindo como ligante quelato, ou seja $\left[\mathrm{Fe}(\mathrm{HA})\left(\mathrm{H}_{2} \mathrm{O}\right)_{4}\right]^{2+}$ e $\left[\mathrm{Fe}\left(\mathrm{H}_{2} \mathrm{~A}\right)\left(\mathrm{H}_{2} \mathrm{O}\right)_{4}\right]^{3+}$. O modelo proposto é apresentado pela série de reações a seguir ${ }^{42}$ :

$$
\begin{aligned}
& \left.\mathrm{Fe}\left(\mathrm{H}_{2} \mathrm{O}\right)_{6}\right]^{3+} \rightleftharpoons\left[\mathrm{Fe}\left(\mathrm{H}_{2} \mathrm{O}\right)_{5} \mathrm{OH}\right]^{2+}+\mathrm{H}^{+} \\
& \mathrm{H}_{2} \mathrm{~A} \rightleftharpoons \mathrm{HA}^{-}+\mathrm{K}^{+} \\
& {\left[\mathrm{Fe}\left(\mathrm{H}_{2} \mathrm{O}\right)_{6}\right]^{3+}+\mathrm{HA}^{-} \rightleftharpoons[\mathrm{Fe}(\mathrm{HA})]^{2+}} \\
& {\left[\mathrm{Fe}\left(\mathrm{H}_{2} \mathrm{O}\right)_{5} \mathrm{OH}\right]^{2+}+\mathrm{H}_{2} \mathrm{~A} \rightleftharpoons[\mathrm{Fe}(\mathrm{HA})]^{2+}} \\
& {\left[\mathrm{Fe}\left(\mathrm{H}_{2} \mathrm{O}\right)_{6}\right]^{2+}+\mathrm{H}_{2} \mathrm{~A} \rightleftharpoons\left[\mathrm{Fe}\left(\mathrm{H}_{2} \mathrm{~A}\right)\right]^{3+}} \\
& {\left[\mathrm{Fe}\left(\mathrm{H}_{2} \mathrm{~A}\right)\right]^{3+} \rightleftharpoons[\mathrm{Fe}(\mathrm{HA})]^{2+}+\mathrm{H}^{+}}
\end{aligned}
$$

A etapa lenta da reação foi encontrada como sendo uma reação de transferência de elétrons por esfera externa com a participação das espécies $\left[\mathrm{Fe}\left(\mathrm{H}_{2} \mathrm{O}\right)_{6}\right]^{3+} \mathrm{e}\left[\mathrm{Fe}\left(\mathrm{H}_{2} \mathrm{O}\right)_{5} \mathrm{OH}\right]^{2+}$, que é inibida pela presença de ferro(II). Como $\mathrm{K}_{\mathrm{a}}=2 \times 10^{-3} \mathrm{~mol} \mathrm{~L}^{-1}\left(25^{\circ} \mathrm{C}\right.$ e força iônica $1 \mathrm{~mol} \mathrm{~L}^{-1}$ ) é bem maior que $\mathrm{K}_{1}=8 \times 10^{-5} \mathrm{~mol} \mathrm{~L} \mathrm{~L}^{-1}$, a desprotonação da espécie $\left[\mathrm{Fe}\left(\mathrm{H}_{2} \mathrm{O}\right)_{6}\right]^{3+}$ deve ocorrer em maior extensão do que com o $\mathrm{H}_{2} \mathrm{~A}$ como função da diminuição da concentração de $\mathrm{H}^{+}$. Assim, os autores registraram que a espécie $\left[\mathrm{Fe}\left(\mathrm{H}_{2} \mathrm{O}\right)_{5} \mathrm{OH}\right]^{2+}$ é mais reativa do que $\left[\mathrm{Fe}\left(\mathrm{H}_{2} \mathrm{O}\right)_{6}\right]^{3+} 42$.

Diferenças na velocidade de reação de oxidação do ácido ascórbico por $\left[\mathrm{Fe}\left(\mathrm{H}_{2} \mathrm{O}\right)_{6}\right]^{3+}$ e $\left[\mathrm{Fe}\left(\mathrm{H}_{2} \mathrm{O}\right)_{5} \mathrm{OH}\right]^{2+}$ também foram observadas nos estudos realizados por Bänsch et alii ${ }^{19}$ em condições de excesso de $\mathrm{H}_{2} \mathrm{~A}$ (cerca de dez vezes) em relação a íons $\mathrm{Fe}(\mathrm{III})$. Uma maior constante de velocidade, de cerca de $5 \times 10^{2}$ vezes, para hidroxos, comparada a de aquo complexos de $\mathrm{Fe}$ (III) (Tabela 2), foi explicada pelos diferentes mecanismos de tranferência de elétrons envolvendo essas duas espécies. A oxidação do $\mathrm{H}_{2} \mathrm{~A}$ por $\left[\mathrm{Fe}\left(\mathrm{H}_{2} \mathrm{O}\right)_{6}\right]^{3+}$, que é menos lábil, segue um mecanismo de transferência de elétrons por esfera externa, enquanto que a oxidação por $\left[\mathrm{Fe}\left(\mathrm{H}_{2} \mathrm{O}\right)_{5} \mathrm{OH}\right]^{2+}$ segue um mecanismo de esfera interna ${ }^{19}$.

$\mathrm{O}$ fato de o íon $\left[\mathrm{Fe}\left(\mathrm{H}_{2} \mathrm{O}\right)_{6}\right]^{3+}$ ser a forma predominante de ferro(III) em meio mais ácido, somado à sua baixa reatividade comparada com $\left[\mathrm{Fe}\left(\mathrm{H}_{2} \mathrm{O}\right)_{5} \mathrm{OH}\right]^{2+}$, faz com que essas duas espécies sejam importantes para estudos cinéticos das reações de ferro(III) na ausência de outros ligantes ${ }^{45}$.

Até aqui foi dada atenção às reações envolvendo apenas aquocomplexos de ferro(III) e, como apresentado na tabela 2, a presença de outros ligantes influenciam fortemente a velocidade de reação do ferro(III) com o ácido ascórbico. A seguir as reações de alguns desses complexos serão abordados com maiores detalhes.

Outras evidências para a formação de complexos intermediários entre íons $\mathrm{Fe}$ (III) e ácido ascórbico têm sido publicadas. Nos estudos de Keypour et alii $^{43}$ foi observada a formação de duas espécies de vida curta em $\mathrm{pH} 2$ durante a reação de cloreto 
de Fe(III) com ácido ascórbico. Eles determinaram uma das espécies como sendo $[\mathrm{FeHA}]^{2+}$, e a outra foi admitida como sendo um complexo de $\mathrm{Fe}$ (II) com o radical ascorbato ${ }^{43}$.

Nos estudos da reação do tris(oxalato)ferrato(III) com ácido ascórbico, Martinez et alii ${ }^{22}$ observaram uma espécie de vida curta, considerada um complexo misto de Fe(III)-oxalato-ascorbato. A partir dos dados cinéticos para a formação da espécie intermediária, os autores descreveram um processo controlado pela reação de substituição de ligantes, a qual é inversamente proporcional à concentração de íons $\mathrm{H}^{+}$. Segue-se uma etapa relativamente lenta de transferência de elétrons por esfera interna, que é independente do $\mathrm{pH}$, da concentração do ácido ascórbico e da força iônica ${ }^{22}$.

Muitos dos sistemas estudados envolvendo Fe(III)-ácido ascórbico apresentam mecanismo de transferência de elétrons por esfera interna. Contudo trabalhos com 2,2'-bipiridina e 1,10fenantrolina e seus derivados como ligantes ao Fe(III) apresentam mecanismo de transferência de elétrons por esfera externa ${ }^{32,33,47 .}$

Bänsch et alii ${ }^{20}$ realizaram estudos cinéticos da oxidação do ácido ascórbico pelo íon complexo $\left[\mathrm{Fe}(\mathrm{CN})_{6}\right]^{3-}$ em solução aquosa como função do $\mathrm{pH}$, da temperatura e da pressão. Os estudos foram realizados em condições de pseudo-primeira ordem com a concentração de ácido ascórbico vinte vezes maior em relação a de íons $\mathrm{Fe}(\mathrm{III})$, em uma faixa de $\mathrm{pH}$ entre 0 e 7 e força iônica $1 \mathrm{~mol} \mathrm{~L}{ }^{-1}$ a $25^{\circ} \mathrm{C}$. Os resultados indicaram que uma constante de velocidade limite é atingida em $\mathrm{pH} \leq 1,0$, isto é, onde está presente unicamente a espécie $\mathrm{H}_{2} \mathrm{~A}^{20}$.

$\mathrm{Na}$ região entre $5,0 \leq \mathrm{pH} \leq 5,3$ a velocidade da reação é independente da acidez do meio, o que foi atribuído à reação redox principalmente associada com a espécie $\mathrm{HA}^{-}$, considerada a espécie predominante nessas condições. Com o $\mathrm{pH}$ próximo a 7 , observou-se um aumento significativo do valor de $\mathrm{k}_{\mathrm{obs}}$, atribuído à presença da espécie $\mathrm{A}^{2-}$ que começa a se formar nessas condições. Os autores concluiram que a reação de redução do complexo $\left[\mathrm{Fe}(\mathrm{CN})_{6}\right]^{3-}$ ocorre por um mecanismo de transferência de elétrons por esfera externa ${ }^{20}$.

Nos estudos da reação de oxidação do ácido ascórbico pelo complexo dimérico $\left[\mathrm{Fe}_{2}(\mathrm{CN})_{10}\right]^{4-}$, foi proposto que a reação ocorre em três estágios, com a redução dos dois íons centrais $\mathrm{Fe}(\mathrm{III})$. O primeiro estágio é a redução de um íon $\mathrm{Fe}$ (III) para formar um dímero composto de Fe(III,II), em seguida ocorre a redução do outro íon Fe(III) formando um dímero de Fe(II). No terceiro estágio foi observado uma pequena variação de absorbância que não foi estudada em detalhes ${ }^{46}$.

Nesses estudos, os mecanismos para ambos os estágios foram considerados similares e envolvem a formação de um íon do tipo $\left[\mathrm{Fe}_{2}(\mathrm{CN})_{10}\right]^{\mathrm{n}-} \cdot \mathrm{M}^{+} \cdot \mathrm{A}^{\mathrm{p}-}$ onde $\mathrm{n}=4$ ou $5, \mathrm{~A}^{\mathrm{p}-}$ é a espécie reagente ascorbato com $\mathrm{p}=1$ ou 2 e $\mathrm{M}^{+}=\mathrm{Li}^{+}, \mathrm{Na}^{+}$ou $\mathrm{K}^{+}$. Foram calculadas as constantes de velocidade de pseudo-segunda ordem $6,44 \times 10^{3}, 2,48 \times 10^{9}$ e $1,25 \times 10^{3}, 2,98 \times 10^{8} \mathrm{~mol}^{-1} \mathrm{~L} \mathrm{~s}^{-1}$ a $19^{\circ} \mathrm{C}$ para as espécies $\mathrm{HA}^{-}$e $\mathrm{A}^{2-}$ para o primeiro e segundo estágios, respectivamente. A reação é afetada por cátions alcalinos, aumentando a velocidade com o aumento do tamanho do íon metálico: $\mathrm{Li}^{+}<\mathrm{Na}^{+}<\mathrm{K}^{+} 46$.

A dificuldade de interpretação dos resultados envolvendo reações com $\mathrm{Fe}(\mathrm{III})$ em amplos intervalos de $\mathrm{pH}$ e em presença de possíveis ligantes, é devido a uma grande variedade de complexos de ferro(III) que podem estar presentes. Podendo-se incluir hidroxo complexos com a ocorrência de formação de precipitados em altos valores de $\mathrm{pH}^{42}$. Outro exemplo, são os resultados apresentados por Hynes e Kelly ${ }^{48}$ mostrando que a decomposição de $[\mathrm{FeCl}]^{+}$é uma complicação a mais na reação de cloreto de ferro(III) com ácido ascórbico.

\subsection{Reações de oxidação do ácido ascórbico por complexos de Co(III)}

Reações redox envolvendo complexos de Co(III), cineticamente inertes, têm apresentado importantes oportunidades para o estudo de mecanismos de reações de transferência de elétrons por esfera externa e interna em complexos de íons metálicos. Em muitos casos não existe a formação do complexo de $\mathrm{Co}$ (III) com o ácido ascórbico ou o ascorbato, uma vez que a reação redox é muito mais rápida ${ }^{8,9,23,26-28}$.

A reação de redução do íon hexaaquocobalto(III) pelo ácido ascórbico foi estudada em meio de alta concentração de íons hidrogênio $\left(0,6-3,0 \mathrm{~mol} \mathrm{~L}{ }^{-1}\right)$, onde apenas a espécie $\mathrm{H}_{2} \mathrm{~A}$ está presente como redutora, e nessas condições ambas as espécies $[\mathrm{Co}(\mathrm{OH})(\mathrm{aq})]^{2+}$ e $[\mathrm{Co}(\mathrm{aq})]^{3+}$ foram consideradas. O mecanismo da reação foi esquematizado de acordo com a sequência de reações apresentadas a seguir ${ }^{8}$ :

$$
\begin{aligned}
& {[\mathrm{Co}(\mathrm{aq})]^{3+} \rightleftharpoons[\mathrm{Co}(\mathrm{OH})(\mathrm{aq})]^{2+}+\mathrm{H}^{+}(\mathrm{aq})} \\
& {[\mathrm{Co}(\mathrm{aq})]^{3+}+\mathrm{H}_{2} \mathrm{~A} \rightarrow \mathrm{Co}^{2+}+\mathrm{H}_{2} \mathrm{~A}^{\bullet}} \\
& {[\mathrm{Co}(\mathrm{OH})(\mathrm{aq})]^{2+}+\mathrm{H}_{2} \mathrm{~A} \rightarrow \mathrm{Co}^{2+}+\mathrm{H}_{2} \mathrm{~A}^{\bullet}} \\
& {[\mathrm{Co}(\mathrm{aq})]^{3+}+\mathrm{H}_{2} \mathrm{~A}^{\bullet} \rightarrow \mathrm{Co}^{2+}+\mathrm{A}_{\mathrm{a} x}+\mathrm{H}^{+} \quad \text { rápida }} \\
& {[\mathrm{Co}(\mathrm{OH})(\mathrm{aq})]^{2+}+\mathrm{H}_{2} \mathrm{~A}^{\bullet} \rightarrow \mathrm{Co}^{2+}+\mathrm{A}_{\mathrm{ox}} \quad \text { rápida }}
\end{aligned}
$$

Mesmo em altas concentrações de íons hidrogênio, usadas por Rickman et alii $^{8}$, a reação é rápida e foi seguida por técnicas de parada instantânea de fluxo ("stopped flow"). Não foi evidenciada a formação de um complexo intermediário, e a reação de transferência de elétrons foi atribuída como sendo um mecanismo de esfera externa ${ }^{8}$.

Estudos cinéticos da reação de redução de alguns complexos de íon cobalto por ácido ascórbico em meio de tampão acetato ( $\mathrm{pH}$ entre 3,6 e 5,0) mostraram que tanto para $\left[\mathrm{Co}(\mathrm{phen})_{3}\right]^{3+}$ como $\left[\mathrm{Co}(\text { bipy })_{3}\right]^{3+}$ a reação é aproximadamente $10^{7}$ vezes mais rápida na presença da espécie $\mathrm{A}^{2-}$ em relação à espécie $\mathrm{HA}^{-}$ (Tabela 2).

$\mathrm{O}$ complexo $\left[\mathrm{Co}(\mathrm{en})(\mathrm{phen})_{2}\right]^{3+}$ é reduzido apenas pela espécie $\mathrm{A}^{2-}$, enquanto que os complexos $\left[\mathrm{Co}(\mathrm{en})_{2}(\mathrm{phen})\right]^{3+} \mathrm{e}$ $\left[\mathrm{Co}(\mathrm{en})_{3}\right]^{3+}$ não são reduzidos por íons ascorbato. Nenhum desses complexos é reduzido pela espécie $\mathrm{H}_{2} \mathrm{~A}$.

Os autores explicaram as diferentes reatividades pela correspondência aos potenciais de redução desses complexos $(0,391$ $\mathrm{V}$ para $\left[\mathrm{Co}(\text { phen })_{3}\right]^{3+/ 2+}, 0,316 \mathrm{~V}$ para $\left[\mathrm{Co}(\text { bipy })_{3}\right]^{3+/ 2+} \mathrm{e}-0,214$ $\mathrm{V}$ para $\left[\mathrm{Co}(\mathrm{en})_{3}\right]^{3+/ 2+}$, que não explicam por que o complexo [Co(EDTA) $]^{-}$não é reduzido pelo ácido ascórbico apesar de seu alto potencial redox $(0,60 \mathrm{~V})^{25}$.

A reação de oxidação do $\mathrm{H}_{2} \mathrm{~A}$ por $\left[\mathrm{Co}\left(\mathrm{C}_{2} \mathrm{O}_{4}\right)_{3}\right]^{3-}$ foi estudada em meio ácido, $3 \leq \mathrm{pH} \leq 5^{28}$, e em meio alcalino, $8 \leq \mathrm{pH} \leq$ $10^{23}$. Nos dois estudos a reação global foi representada por:

$\mathrm{H}_{2} \mathrm{~A}+2\left[\mathrm{Co}\left(\mathrm{C}_{2} \mathrm{O}_{4}\right)_{3}\right]^{3-} \rightarrow \mathrm{A}_{6 \mathrm{x}}+2 \mathrm{H}^{+}+2\left[\mathrm{Co}\left(\mathrm{C}_{2} \mathrm{O}_{4}\right)_{3}\right]^{4}$

Os dados cinéticos foram quantitativamente analisados, sugerindo um mecanismo de transferência de elétrons por esfera externa com formação do radical intermediário $\mathrm{A}^{-1}$ na etapa determinante da velocidade da reação ${ }^{23}$.

Nesses trabalhos concluiu-se que a reação (eq. 23) é extremamente dependente do $\mathrm{pH}$, sendo a espécie $\mathrm{A}^{2-}$ (ânion ascorbato) mais reativa em comparação com as espécies protonadas $\mathrm{H}_{2} \mathrm{~A}$ e $\mathrm{HA}^{-23,28}$.

Os estudos da reação de oxidação do $\mathrm{H}_{2} \mathrm{~A}$ por $\left[\mathrm{Co}\left(\mathrm{C}_{2} \mathrm{O}_{4}\right)_{3}\right]^{3-}$ em uma ampla faixa de $\mathrm{pH}$ (3-10) em meio de tampão universal onde foram combinados dados cinéticos $\left(\log \mathrm{k}_{\mathrm{obs}} v s \mathrm{pH}\right)$ com o diagrama de distribuição do ácido ascórbico confirmam a ordem de reatividade $\mathrm{H}_{2} \mathrm{~A}<\mathrm{HA}^{-}<\mathrm{A}^{2-}(\text { Fig. 4) })^{29}, 30$.

Os estudos do efeito catalítico de íons ferro e da acidez na reação de oxidação do $\mathrm{H}_{2} \mathrm{~A}$ por $\left[\mathrm{Co}\left(\mathrm{C}_{2} \mathrm{O}_{4}\right)_{3}\right]^{3-}$ foram realizados em presença de excesso de EDTA, para evitar a hidrólise de íons ferro e precipitação do oxalato de $\mathrm{Co}(\mathrm{II})$, facilmente observados em $\mathrm{pH}>829,30$.

$\mathrm{Na}$ figura 4 observa-se que para $\mathrm{pH}<5$, onde as espécies 


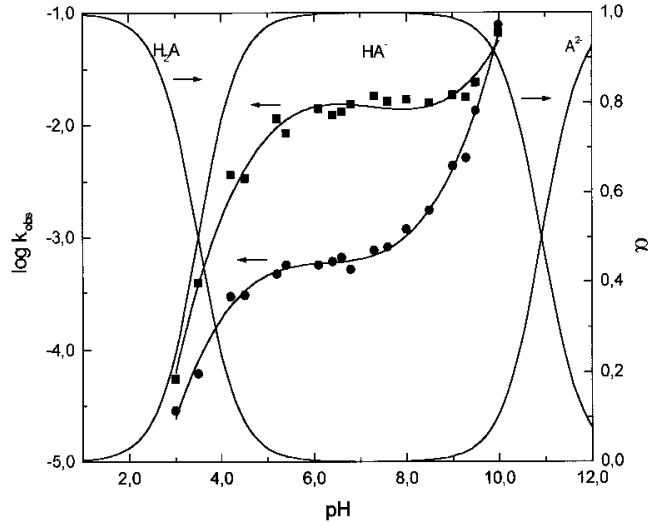

Figura 4. Coeficiente de distribuição (a) do ácido L-ascórbico, $K_{1}=3,2 \times 10^{-4}$ e $K_{2}=1,3 \times 10^{-11} \mathrm{~mol} \mathrm{~L}^{-1}$ como função do $\mathrm{pH}^{6,9}$. Dependência da constante de velocidade com o pH, sem (•) e com [Fe(III)] $E D T A]=1,00 \times 10^{-4} \mathrm{~mol} \mathrm{~L}^{-1}(\boldsymbol{\square}) \cdot\left[\mathrm{H}_{2} \mathrm{~A}\right]=3,00 \times 10^{-2} \mathrm{~mol} \mathrm{~L}^{-1},\left[\mathrm{Co}\left(\mathrm{C}_{2} \mathrm{O}_{4}\right)_{3}\right]^{3-}$

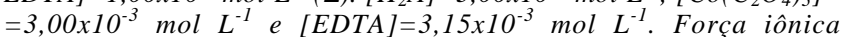
$1,0 \mathrm{~mol} \mathrm{~L}^{-1}(\mathrm{NaCl}),(25,0 \pm 0,1)^{\circ} \mathrm{C}$ em meio de tampão universal ${ }^{29,30}$.

$\mathrm{H}_{2} \mathrm{~A}$ e $\mathrm{HA}^{-}$estão presentes, a reação é mais lenta mesmo em presença do catalisador. Na faixa de $\mathrm{pH}$ entre 5 e 9, o valor de $\mathrm{k}_{\text {obs }}$ para a reação catalisada praticamente independe da acidez do meio, o que é caracterizado por um patamar. Para a reação não catalisada, esse patamar é menor, e o valor de $\mathrm{k}_{\text {obs }}$ aumenta a partir de $\mathrm{pH} 7,5$, quando começa a surgir a espécie $\mathrm{A}^{2-}$ em solução ${ }^{29,30}$.

Os resultados da Figura 4 para a reação não catalisada estão em concordância com a seguinte sequência de reações:

$$
\begin{aligned}
& \mathrm{H}_{2} \mathrm{~A} \rightleftharpoons \mathrm{HA}^{-}+\mathrm{H}^{+} \quad \mathrm{K}_{1} \\
& \mathrm{HA}^{-} \rightleftharpoons \mathrm{A}^{2-}+\mathrm{H}^{+} \\
& \mathrm{H}_{2} \mathrm{~A}+\left[\mathrm{Co}\left(\mathrm{C}_{2} \mathrm{O}_{4}\right)_{3}\right]^{3-} \rightarrow \mathrm{H}_{2} \mathrm{~A}^{+\bullet}+\mathrm{Co}^{2+}+3 \mathrm{C}_{2} \mathrm{O}_{4}{ }^{2-} \quad \mathrm{k}_{\mathfrak{i}} \quad \text { (26) } \\
& \mathrm{HA}^{-}+\left[\mathrm{Co}\left(\mathrm{C}_{2} \mathrm{O}_{4}\right)_{3}\right]^{3-} \rightarrow \mathrm{HA}^{\bullet}+\mathrm{Co}^{2+}+3 \mathrm{C}_{2} \mathrm{O}_{4}{ }^{2-} \quad \mathrm{k}_{\mathrm{h}} \\
& \mathrm{A}^{2-}+\left[\mathrm{Co}\left(\mathrm{C}_{2} \mathrm{O}_{4}\right)_{3}\right]^{3-} \rightarrow \mathrm{A}^{-\bullet}+\left[\mathrm{Co}\left(\mathrm{C}_{2} \mathrm{O}_{4}\right)_{3}\right]^{4-} \quad \mathrm{k}_{\mathrm{c}}
\end{aligned}
$$

\section{REAÇÕES DE OXIDAÇÃO DE ÁCIDO ASCÓRBICO POR ÍONS METÁLICOS DE TRANSIÇÃO CATALISADAS POR ÍONS FERRO}

O efeito catalítico de íons ferro também foi estudado em alguns outros sistemas envolvendo reações redox do ácido ascórbico com peróxido de hidrogênio ${ }^{49}$ e complexos de crômio (IV) ${ }^{31,50}$.

Nos estudos do efeito catalítico de íons ferro, na ausência ou presença de agentes complexantes, tem sido mostrado que o íon ferro é reciclado entre os estados de oxidação di e tripositivos ${ }^{31}$.

Algumas das sequências de reações propostas envolvem a formação de complexos $\mathrm{Fe}$ (III)-ascorbato ou Fe(III)-quelanteascorbato que reagem na etapa determinante da velocidade da reação ${ }^{31,51,52}$.

Um exemplo interessante é a reação lenta de diperoxocromio (IV) derivado do dietilenetriamina, $\mathrm{Cr}(\mathrm{IV})($ dien $)\left(\mathrm{O}_{2}\right)_{2}$, com o acido ascórbico em meio de tampão acetato, a qual é fortemente catalisada por íons de $\mathrm{Fe}$ (III) ou Fe(III), tanto na ausência como na presença de EDTA. Na ausência de EDTA, a reação é de primeira ordem $\left(\mathrm{k}=4 \times 10^{3} \mathrm{~mol}^{-1} \mathrm{~L} \mathrm{~s}^{-1}\right)$ tanto para o $\mathrm{Cr}(\mathrm{IV})$ como para o catalisador (eq. 30$)^{31}$.

A sequência de reações proposta envolve a formação do complexo $\mathrm{Fe}$ (III)-ascorbato, $\mathrm{K}=5 \times 10^{3} \mathrm{~mol}^{-1} \mathrm{~L}$ (eq. 31 ). Esse complexo reage com o oxidante $\mathrm{Cr}(\mathrm{IV})$, na etapa determinante com constante de velocidade de segunda ordem, $\mathrm{k}=1,5 \times 10^{3} \mathrm{~mol}^{-1} \mathrm{~L}$ $\mathrm{s}^{-1}$ (eq. 32), produzindo $\mathrm{Cr}(\mathrm{III})$ e o radical ascorbato $\left(\mathrm{HA}^{\Uparrow}\right)$, e este último rapidamente reage com $\mathrm{Cr}(\mathrm{IV})$ (eq. 33). As reações que seguem são todas muito rápidas (eq. 33-35) ${ }^{31}$.

$$
\begin{aligned}
& \mathrm{Fe}(\mathrm{II})+\mathrm{Cr}(\mathrm{IV})\left(\mathrm{O}_{2}\right)_{2} \rightarrow \mathrm{Fe}(\mathrm{III})+\mathrm{Cr}(\mathrm{III})\left(\mathrm{O}_{2}\right)_{2}^{-} \\
& \mathrm{Fe}(\mathrm{III})+\mathrm{HA}^{-} \rightarrow \mathrm{Fe}(\mathrm{III})\left(\mathrm{HA}^{-}\right) \\
& \mathrm{Fe}(\mathrm{III})\left(\mathrm{HA}^{-}\right)+\mathrm{Cr}(\mathrm{IV})\left(\mathrm{O}_{2}\right)_{2} \rightarrow \mathrm{Fe}(\mathrm{III})+\mathrm{HA}^{-}+\mathrm{Cr}(\mathrm{III})\left(\mathrm{O}_{2}\right)_{2}^{-} \\
& \mathrm{HA}^{-}+\mathrm{Cr}(\mathrm{IV})\left(\mathrm{O}_{2}\right)_{2} \rightarrow \mathrm{A}_{0 \mathrm{x}}+\mathrm{Cr}(\mathrm{III})\left(\mathrm{O}_{2}\right)_{2}+\mathrm{H}^{+} \quad \text { rápida } \\
& \mathrm{Fe}(\mathrm{III})+\mathrm{HA}^{-} \rightarrow \mathrm{Fe}(\mathrm{II})+\mathrm{H}^{+}+\mathrm{A}_{6 \mathrm{X}} \\
& \mathrm{Cr}(\mathrm{III})\left(\mathrm{O}_{2}\right)_{2}+2 \mathrm{H}_{2} \mathrm{~A}+\mathrm{H}^{+} \rightarrow \mathrm{Cr}(\mathrm{III})+4 \mathrm{H}_{2} \mathrm{O}+2 \mathrm{~A}_{0 \times} \text { rápida }
\end{aligned}
$$

Os estudos da redução de $\mathrm{Cr}(\mathrm{IV})$ catalisada por íons ferro em presença de EDTA mostraram que em altas concentrações de ácido ascórbico existe a formação de um complexo entre $\mathrm{Fe}$ (III)/EDTA e ascorbato, $\mathrm{K}=88 \mathrm{~mol}^{-1} \mathrm{~L}$ (eq. 36). Em seguida ocorre uma reação de óxido-redução que destrói o complexo originando o radical $\mathrm{HA}^{\Uparrow}$, que é a etapa determinante da velocidade da reação, $\mathrm{k}=3,2 \times 10^{3} \mathrm{~mol}^{-1} \mathrm{~L} \mathrm{~s}^{-1}$ (eq. 37) ${ }^{31}$.

$$
\begin{aligned}
& \mathrm{Fe}(\mathrm{III}) / \mathrm{EDTA}+\mathrm{HA}^{-} \rightleftharpoons \mathrm{Fe}(\mathrm{III})(\mathrm{EDTA})\left(\mathrm{HA}^{-}\right) \\
& \mathrm{Fe}(\mathrm{III})\left(\mathrm{EDTA}^{-}\right)\left(\mathrm{HA}^{-}\right) \rightarrow \mathrm{Fe}(\mathrm{II})(\mathrm{EDTA})+\mathrm{HA}^{\circ}
\end{aligned}
$$

$\mathrm{HA}^{\bullet}+\mathrm{Cr}(\mathrm{IV})\left(\mathrm{O}_{2}\right)_{2} \rightarrow \mathrm{A}_{\mathrm{ox}}+\mathrm{Cr}(\mathrm{III})\left(\mathrm{O}_{2}\right)_{2}^{-}+\mathrm{H}^{+}$rápida (38)

$\mathrm{Fe}(\mathrm{II})(\mathrm{EDTA})+\mathrm{Cr}(\mathrm{IV})\left(\mathrm{O}_{2}\right)_{2} \rightarrow \mathrm{Cr}(\mathrm{III})\left(\mathrm{O}_{2}\right)_{2}{ }^{-}+\mathrm{Fe}(\mathrm{III})(\mathrm{EDTA})$ rápida

As duas sequências de reações anteriores mostram um resultado interessante para a reação de Fe(III) com o ascorbato: as velocidades de formação dos complexos $\mathrm{Fe}$ (III)-ascorbato ou $\mathrm{Fe}(\mathrm{III}) /$ EDTA-ascorbato são menores do que o processo de transferência de elétrons que resulta na destruição dos mesmos ${ }^{31}$.

As razões entre as concentrações das formas oxidada e reduzida do íon ferro nesses sistemas são dependentes da velocidade específica de dois processos redox. O primeiro envolvendo a oxidação do $\mathrm{Fe}(\mathrm{II})$ representado pelas equações (30 e 39) e o segundo envolvendo a reação de redução do íon $\mathrm{Fe}(\mathrm{III})$ (eq. 34 e 37$)^{31}$

Para a reação da redução de $\mathrm{Cr}(\mathrm{IV})$ por ácido ascórbico catalisada por ferro em ausência ou presença de EDTA foi proposto um mecanismo de transferência de elétrons por esfera interna ${ }^{31}$

Nos estudos da reação de oxidação do ácido ascórbico pelo $\left[\mathrm{Co}\left(\mathrm{C}_{2} \mathrm{O}_{4}\right)_{3}\right]^{3-}$ catalisada por íons ferro em ampla faixa de $\mathrm{pH}$ (Fig. 4) observa-se que o efeito catalítico é acentuado em condições onde predomina a espécie $\mathrm{HA}^{-}$, faixa de $\mathrm{pH}$ entre 5 e 8. Esse efeito é explicado pela formação do complexo entre $\mathrm{Fe}(\mathrm{III}) /$ EDTA e ascorbato (eq. 40), que é rapidamente destruído devido a reações redox com a formação do radical $\mathrm{HA}^{\Uparrow}{ }^{29,30}$.

Para a reação catalisada em presença de excesso de EDTA o mecanismo pode ser representado pela sequência de reações 40 a $44^{29,30}$ :

$$
\begin{aligned}
& \mathrm{Fe}(\mathrm{III})(\mathrm{EDTA})+\mathrm{HA}^{-} \rightleftharpoons \mathrm{Fe}(\mathrm{III})\left(\mathrm{EDTA}^{-}\left(\mathrm{HA}^{-}\right)\right. \\
& \mathrm{Fe}(\mathrm{III})\left(\mathrm{EDTA}^{-}\left(\mathrm{HA}^{-}\right) \rightarrow \mathrm{Fe}(\mathrm{II})\left(\mathrm{EDTA}^{-}\right)+\mathrm{HA}^{\bullet}\right.
\end{aligned}
$$

$\mathrm{Fe}(\mathrm{II}) \mathrm{EDTA}+\left[\mathrm{Co}\left(\mathrm{C}_{2} \mathrm{O}_{4}\right)_{3}\right]^{3 \cdot} \rightarrow \mathrm{Fe}(\mathrm{III}) \mathrm{EDTA}+\mathrm{Co}^{2+}+3 \mathrm{C}_{2} \mathrm{O}_{4}{ }^{2-}(42)$

$\mathrm{HA}^{\bullet}+\left[\mathrm{Co}\left(\mathrm{C}_{2} \mathrm{O}_{4}\right)_{3}\right]^{3-} \rightarrow \mathrm{A}_{4, \mathrm{x}}+\mathrm{Co}^{2+}+3 \mathrm{C}_{2} \mathrm{O}_{4}{ }^{2-}$

$\mathrm{Fe}(\mathrm{III}) \mathrm{EDTA}+\mathrm{HA}^{*} \rightarrow \mathrm{Fe}(\mathrm{II}) \mathrm{EDTA}+\mathrm{A}_{\mathrm{ux}}+\mathrm{H}^{+}$

Pela sequência de reações $40-44$ conclui-se que a reação de transferência de elétrons entre $\mathrm{Co}(\mathrm{III})$ e $\mathrm{Fe}(\mathrm{II})$ é muito mais 
rápida do que a reação do complexo de Co(III) com o ácido ascórbico, e a reação de $\mathrm{Fe}(\mathrm{III})$ com ácido ascórbico é sempre muito rápida, como já apresentado na tabela 2 .

\section{REAÇÕES DE OXIDACCÃO DO ÁCIDO ASCÓRBICO POR OXIGÊNIO CATALISADAS POR ÍONS METÁLICOS}

Muitos dos trabalhos realizados sobre reações catalisadas dizem respeito à chamada "autoxidação" do ácido ascórbico, isto é, o processo de oxidação do ácido ascórbico pelo oxigênio dissolvido em soluções aquosas. Os cátions metálicos normalmente usados como catalisadores na oxidação do ácido ascórbico são $\mathrm{Cu}^{2+}, \mathrm{Fe}^{3+}, \mathrm{Ni}^{2+}$ e $\mathrm{Co}^{3+}$, sendo que apenas o potencial de redução dessas espécies não é suficiente para explicar a seletividade e habilidade específica para esse efeito catalítico ${ }^{53}$.

A maioria dos estudos cinéticos envolvendo reações de autoxidação do ácido ascórbico envolvem a catálise por íons cobre. Essas reações são de grande interesse por serem usadas em sistemas bioquímicos como modelos para metaloenzimas oxidases que contêm cobre(II), como por exemplo ascorbato oxidase, polifenol oxidase e ceruloplasmina ${ }^{51,52,54-57}$.

Desses estudos o mecanismo geral proposto apresenta as seguintes etapas:

a) formação de um complexo ascorbato-cobre(II),

b) transferência de carga a partir do ligante (ascorbato) para o metal produzindo a espécie $\mathrm{Cu}(\mathrm{I})$ e

c) rápida reoxidação do $\mathrm{Cu}(\mathrm{I})$ pelo oxigênio molecular regenerando o catalisador ${ }^{51,52,55,56,58}$.

Alguns estudos também propõe um mecanismo de reação em cadeia com a transferência de dois elétrons para o oxigênio, tendo como espécie reativa complexos diméricos de cobreascorbato, com a participação efetiva da espécie $\mathrm{Cu}$ (III) no ciclo catalítico, representado no esquema de reações a seguir (eq. 45$53)^{54,59}$

Início:

$2 \mathrm{Cu}^{2+}{ }_{(\mathrm{all})}+\mathrm{HA}^{-} \rightarrow\left[\mathrm{Cu}_{2} \mathrm{~A}\right]^{2+}+\mathrm{H}^{+}$

$\left[\mathrm{Cu}_{2} \mathrm{~A}\right]^{2+}+\mathrm{O}_{2} \rightleftharpoons\left[\mathrm{Cu}_{2} \mathrm{~A}\left(\mathrm{O}_{2}\right)\right]^{2+}$

$\left[\mathrm{Cu}_{2} \mathrm{~A}\left(\mathrm{O}_{2}\right)\right]^{2+} \rightarrow\left[\mathrm{CuA}\left(\mathrm{O}_{2}\right)\right]^{\bullet}+\mathrm{Cu}^{3+}(\mathrm{aq})$

$\mathrm{Cu}^{3+}(\mathrm{aq})+\mathrm{H}_{2} \mathrm{O} \rightleftharpoons \mathrm{Cu}^{2+}(\mathrm{aq})+\mathrm{OH}^{*}+\mathrm{H}^{+}$

$\mathrm{OH}^{\bullet}+\mathrm{H}_{2} \mathrm{~A} \rightarrow \mathrm{A}^{\bullet}+\mathrm{H}_{3} \mathrm{O}^{+}$

(ou $\mathrm{OH}^{\bullet}+\mathrm{HA}^{-} \rightarrow \mathrm{A}^{\bullet}+\mathrm{H}_{2} \mathrm{O}$ em $\mathrm{pH}$ mais alto

Propagação:

$\mathrm{A}^{-\bullet}+\left[\mathrm{Cu}_{2} \mathrm{~A}\left(\mathrm{O}_{2}\right)\right]^{2+} \rightarrow\left[\mathrm{CuA}\left(\mathrm{O}_{2}\right)\right]^{-\bullet}+\mathrm{A}_{\mathrm{i}) \mathrm{X}}+\mathrm{Cu}^{2+}$

$\left[\mathrm{CuA}\left(\mathrm{O}_{2}\right)\right]^{-\bullet} \rightarrow \mathrm{Cu}^{2+}(\mathrm{aq})+\mathrm{A}^{-\bullet}+\mathrm{O}_{2}^{2-}$

Término:

$2\left[\mathrm{CuA}\left(\mathrm{O}_{2}\right)\right]^{-\bullet} \rightarrow 2 \mathrm{O}_{2}^{2-}+\mathrm{A}^{2-\bullet}+\mathrm{A}_{\mathrm{ox}}+2 \mathrm{Cu}^{2+}(\mathrm{aq})$

O consumo de oxigênio durante a reação de oxidação de ácido ascórbico catalisada por íons cobre(II) foi monitorado amperometricamente com eletrodo seletivo de $\mathrm{O}_{2}$ e espectrofluorimetricamente através do efeito supressor do $\mathrm{O}_{2}$ na intensidade de radiação emitida pelo complexo $\left[\mathrm{Ru}(\text { bipy })_{3}\right]^{2+56}$.

Sasso et alii $^{56}$ realizaram estudos em solução, saturada com oxigênio, com concentração inicial de ácido ascórbico $\left(4,13 \times 10^{-2} \mathrm{~mol}\right.$ $\left.\mathrm{L}^{-1}\right), \mathrm{KNO}_{3} 0,10 \mathrm{~mol} \mathrm{~L}^{-1}, \mathrm{pH} \mathrm{2,17}\left(\mathrm{HNO}_{3}\right)$ e concentração de íons $\mathrm{Cu}(\mathrm{II})$ entre $(0,05-0,4) \times 10^{-3} \mathrm{~mol} \mathrm{~L} \mathrm{~L}^{-1}$ a $25^{\circ} \mathrm{C}$. Observou-se que a velocidade do consumo de oxigênio é independente da concentração inicial de $\left[\mathrm{Ru}(\mathrm{bipy})_{3}\right]^{2+}\left(3-30 \mu \mathrm{mol} \mathrm{L}^{-1}\right)$ e é de meia-ordem com respeito a concentração de oxigênio ${ }^{56}$. A constante de velocidade de pseudo-meia ordem observada foi $46\left(\mathrm{~mol} \mathrm{~L}^{-1}\right)^{-1 / 2} \mathrm{~min}^{-}$ 1 , semelhante ao valor $48\left(\mathrm{~mol} \mathrm{~L}^{-1}\right)^{-1 / 2} \mathrm{~min}^{-1}$ obtido nos estudos de Jameson e Blackburn ${ }^{59}$.

Estudos cinéticos comparando o efeito catalítico de íons $\mathrm{Cu}(\mathrm{II})$ e $\mathrm{Fe}(\mathrm{III})$ na oxidação do ácido ascórbico pelo oxigênio molecular, em meio ácido ( $\mathrm{pH} 2$ - 5,5), mostraram que a atividade catalítica do íon $\mathrm{Cu}$ (II) é maior que a do íon Fe(III) para a autoxidação da espécie $\mathrm{HA}^{-}$. Porém para a oxidação da forma protonada, $\mathrm{H}_{2} \mathrm{~A}$, o íon $\mathrm{Fe}(\mathrm{III})$ teve efeito catalítico melhor do que o íon $\mathrm{Cu}(\mathrm{II})$. Em meio contendo agente quelante essa ordem catalítica pode ser diferente, sendo também função da acidez do meio $^{51}$.

A formação de um complexo do íon metálico com o ácido ascórbico, na etapa de pré-equilíbrio, explica a dependência da velocidade da reação em relação a acidez do meio e a maior reatividade do íon metálico com o ânion monoprotonado, $\mathrm{HA}^{-}$, do que com a forma protonada, $\mathrm{H}_{2} \mathrm{~A}^{51}$.

A diferença das reatividades dos íons $\mathrm{Cu}$ (II) e $\mathrm{Fe}$ (III) para a oxidação das espécies $\mathrm{HA}^{-}$e $\mathrm{H}_{2} \mathrm{~A}$ é explicada como função das diferentes constantes de formação de complexos dos íons metálicos com essas duas espécies. Para a oxidação da forma $\mathrm{H}_{2} \mathrm{~A}$ existe maior probabilidade de formação do complexo $\mathrm{FeH}_{2} \mathrm{~A}^{3+}$ comparado com $\mathrm{CuH}_{2} \mathrm{~A}^{2+}$ devido à maior carga do íon férrico ${ }^{51}$.

Taqui Khan e Martell ${ }^{52}$ também estudaram o efeito catalítico de íons quelatos de $\mathrm{Cu}(\mathrm{II})$ e $\mathrm{Fe}(\mathrm{III})$ na reação de oxidação do ácido ascórbico pelo oxigênio molecular, em meio ácido $(\mathrm{pH}$ $2,25-3,45)$. Os autores observaram que para quelatos de um mesmo íon metálico a velocidade diminui com o aumento da estabilidade e da carga negativa do composto metal-quelato.

As atividades catalíticas dos quelatos de cobre(II) diminuíram na seguinte ordem: iminodiacetato de cobre(II), $\mathrm{Cu}$ (II)-IMDA > 2hidroxietilimino-diacetato de cobre(II), $\mathrm{Cu}(\mathrm{II})$-HIMDA > etilenodiaminotetracetato de cobre(II), Cu(II)-EDTA ${ }^{52}$.

As atividades catalíticas dos quelatos de ferro(III) diminuíram na seguinte ordem: 2-hidroxietiliminodiacetato de ferro(III), $\mathrm{Fe}$ (III)-HIMDA > nitrilotriacetato de ferro(III), Fe(III)-NTA > 2-hidroxietilenodiaminotriacetato de ferro(III), Fe(III)-HEDTA $>$ etilenodiaminotetracetato de ferro(III), Fe(III)-EDTA > ciclohexanodiaminotetracetato de ferro(III), Fe(III)-CDTA > dietilenotriamino-pentacetato de ferro(III), Fe(III)-DTPA ${ }^{52}$.

As atividades catalíticas dos quelatos tanto de cobre(II) como de ferro(III) foram independentes da concentração de oxigênio. Foi encontrada uma relação linear entre a estabilidade do composto metal-quelato e sua atividade catalítica. As diferenças nas reatividades dos vários compostos metal-quelato estudadas foram interpretadas com base nas energias de reação ${ }^{52}$.

Estudos em presença de quelatos mostraram que a velocidade da reação de autoxidação do ácido ascórbico catalisada por compostos quelatos de $\mathrm{Fe}(\mathrm{III})$ é dez vezes mais rápida do que por compostos quelatos de $\mathrm{Cu}(\mathrm{II})$; por exemplo, em pH 3,45 a constante de velocidade para Fe-EDTA é $1,0 \times 10^{2} \mathrm{~mol}^{-1} \mathrm{~L} \mathrm{~s}^{-1}$ e para Cu-EDTA é $1,2 \times 10^{1} \mathrm{~mol}^{-1} \mathrm{~L} \mathrm{~s}^{-1} 52$.

O mecanismo proposto pelos autores é uma participação direta da espécie metal-quelato no processo de transferência de elétrons. Numa etapa de pré-equilíbrio há a formação da espécie $\mathrm{MLHA}^{(\mathrm{n}-1)+}$ (eq. 55), sendo a etapa determinante a transferência do elétron a partir do ascorbato para o íon metálico gerando a espécie $\mathrm{MLHA}^{\Uparrow(\mathrm{n}-1)+}$ (eq. 56). Essa última espécie se dissocia em ML $\mathrm{ML}^{(\mathrm{n}-1)+} \mathrm{e} \mathrm{A}^{-\Uparrow}$ (eq. 57) que são oxidados em etapas rápidas pelo $\mathrm{O}_{2}$ do ar, dissolvido na solução ${ }^{52}$.

Assim, a velocidade da reação não depende da concentração do oxigênio, pois esse participa apenas de etapas rápidas. $\mathrm{O}$ mecanismo pode ser ilustrado pela sequência de reações abaixo ${ }^{52}$ :

$\mathrm{H}_{2} \mathrm{~A} \rightleftharpoons \mathrm{HA}^{-}+\mathrm{H}^{+} \quad \mathrm{K}_{1}$

, $\mathrm{HA}^{-}+\mathrm{ML}^{\mathrm{n}+} \rightleftharpoons \mathrm{MLHA}^{(\mathrm{n}-1)+} \rightleftharpoons \mathrm{K}_{2}$ 
$\mathrm{MLHA}^{(\mathrm{n}-1)+} \rightarrow \mathrm{MLHA}^{\cdot(\mathrm{n}-1)+}$

$\mathrm{MLHA}^{\bullet(\mathrm{n}-1)+} \rightarrow \mathrm{A}^{-}+\mathrm{ML}^{(\mathrm{n}-\mathrm{I})+}+\mathrm{H}^{+} \quad$ rápida

$\mathrm{A}^{*}+\mathrm{ML}^{\mathrm{n}+} \rightarrow \mathrm{A}_{\mathrm{bx}}+\mathrm{ML}^{(\mathrm{n}-1)+} \quad$ rápida

É interessante considerar os estudos de oxidação do ácido ascórbico por íons $\mathrm{Cu}(\mathrm{II})$, realizados na ausência de oxigênio dissolvido em solução. Nesse caso não existe regeneração de $\mathrm{Cu}(\mathrm{II})$, devido à oxidação de $\mathrm{Cu}(\mathrm{I})$ pelo oxigênio, não havendo portanto um efeito catalítico de íons cobre na autoxidação de ácido ascórbico ${ }^{24}$.

$\mathrm{Xu}$ e Jordan ${ }^{24}$ estudaram a reação direta de íons cobre(II) aquoso com ácido ascórbico em ausência de oxigênio, meio $\mathrm{NaClO}_{4} / \mathrm{HClO}_{4} 1 \mathrm{~mol} \mathrm{~L}^{-1}$ sobre condições de $\left[\mathrm{Cu}^{2+}\right]>$ [ácido ascórbico]. Essa última condição foi para garantir apenas a formação de complexos 1:1 de cobre(II)-ascorbato e também minimizar a possível reação de ascorbato com seus produtos de oxidação.

Devido a labilidade do $\mathrm{Cu}(\mathrm{II})$ aquoso os autores propuseram um mecanismo de reação de esfera interna, com a etapa determinante sendo a formação do radical HA ${ }^{\Uparrow}$ com constante de velocidade, $\mathrm{k}=2 \times 10^{-2} \mathrm{~s}^{-1}$. Entretanto, $\mathrm{Cu}$ (II) é um doador em potencial de dois elétrons podendo ocorrer um processo de transferência de dois elétrons dando $\mathrm{Cu}(0)$ que reage com $\mathrm{Cu}^{2+}$ aquoso para produzir $\mathrm{Cu}^{+24}$ :

$\mathrm{Cu}^{2+}+\mathrm{HA}^{-} \rightarrow \mathrm{Cu}+\mathrm{A}_{\mathrm{ux}}+\mathrm{H}^{+}$

$\mathrm{Cu}^{2+}+\mathrm{Cu} \rightleftharpoons 2 \mathrm{Cu}^{+} \mathrm{K} \cong 10^{-6} \mathrm{M}$

Os autores também observaram um forte efeito catalítico de ânions $\mathrm{Cl}^{-}$e acetato para a reação de oxidação, não catalisada, do ácido ascórbico por íons $\mathrm{Cu}$ (II). Esse aumento da velocidade pode ser explicado por esquemas similares de reação para ambos os ânions. Para o $\mathrm{Cl}^{-}$os autores concluíram que o mecanismo de reação pode ser tanto por esfera externa (14 vezes mais rápido do que em ausência de $\left.\mathrm{Cl}^{-}\right)$ou por esfera interna (600 mais rápida do que em presença de $\left.\mathrm{Cl}^{-}\right)^{24}$.

Observaram também que o acetato é 50-100 vezes mais efetivo do que cloreto para catalisar a oxidação do ascorbato pelo $\mathrm{Cu}$ (II). Uma conclusão simplificada é que os ânions reduzem a barreira energética para transformar $\mathrm{Cu}^{2+} \mathrm{em} \mathrm{Cu}^{+}$, explicando a aceleração da velocidade ${ }^{24}$.

No entanto os estudos de $\mathrm{Harel}^{60}$ mostram que o $\mathrm{NaCl}$ inibe a oxidação do ácido ascórbico e as reações que produzem $\mathrm{O}_{2}^{-\Uparrow}$, $\mathrm{H}_{2} \mathrm{O}_{2}$ e $\mathrm{HO}^{\Uparrow}$ por evitar a interação de metais de transição (Fe, $\mathrm{Cu}$ ) com o oxigênio. Se o metal de transição está complexado com um ligante fraco como acetato ou ADP, o $\mathrm{NaCl}$ evita a interação entre o oxigênio e o metal. Mas, se o íon metálico está complexado com um ligante forte como citrato ou EDTA, o efeito do $\mathrm{NaCl}$ é fraco ou não existe ${ }^{60}$.

Estudos realizados por Sarma et alii $^{61}$ mostraram que a autoxidação do ácido ascórbico catalisada por íons $\mathrm{Cu}$ (II) foi evitada pela presença de antocianinas (derivadas de cianidinas). Esse efeito de proteção do ácido ascórbico foi atribuído, não somente a formação de complexos quelatos com íons cobre, mas também a mecanismos envolvendo a formação de complexos mistos de ácido ascórbico-cobre-antocianina ${ }^{61}$.

\section{AGRADECIMENTOS}

Ao CNPq e FAPESP pelo apoio financeiro.

\section{REFERÊNCIAS}

1. Davies, M. B.; Austin, J.; Partridge, D. A.; Vitamin C Its Chemistry and Biochemistry, Royal Society of Chemistry, Cambridge 1991.

2. Davies, M. B.; Polyhedron 1992, 11, 285.
3. Roig, M. G.; Rivera, Z. S.; Kennedy, J. F.; Int. J. Food Sci. Nutr. 1993, 44, 59.

4. Washko, P. W.; Welch, R. W.; Dhariwal, K. R.; Wang, Y.; Levine, M.; Anal. Biochem. 1992, 204, 1.

5. Pachla, L. A.; Reynolds, D. L.; Kissinger, P. T.; J. Assoc. Off. Anal. Chem. 1985, 68, 1.

6. Tur'yan, Y. I.; Kohen, R.; J. Electroanal. Chem. 1995, 380, 273.

7. Abe, Y.; Okada, S.; Horii, H.; Taniguchi, S.; J. Chem. Soc. Perkin Trans. II 1987, 715.

8. Rickman, R. A.; Sorensen, R. L.; Watkins, K. O.; Davies, G.; Inorg. Chem. 1977, 16, 1570.

9. Willians, N. H.; Yandell, K.; Aust. J. Chem. 1982, 35,1133 .

10. Stolyarov, K. P.; Amantova, I. A.; Talanta 1967, 14, 1237.

11. Maslowska, J.; Owczarek, A.; Polish J. Chem. 1995, 69, 1230.

12. Jabs, W.; Gaube, W.; Z. Anorg. Allg. Chem. 1984, 514, 185.

13. Jabs, W.; Gaube, W.; Z. Anorg. Allg. Chem. 1986, 538, 166.

14. Martinez, P.; Uribe, D.; Z. Naturforsch., B: Anorg. Chem., Org. Chem. 1982, 37B, 1446. Apud: Davies, M. B.; Polyhedron 1992, 11, 285.

15. Hollis, L. S.; Amundsen, A. R.; Stern, E. W.; J. Am. Chem. Soc. 1985, 107, 274.

16. Nordenskiöld, L.; Kowalewski, J.; Benetis, N.; Inorg. Chim. Acta 1981, 56, L21.

17. Benetis, N.; Holmén, K.; Kowalewski, J.; Nordenskiöld, L.; Wahlberg, O.; Acta Chem. Scand. 1981, A35, 513.

18. Creutz, C.; Inorg. Chem. 1981, 20, 4449.

19. Bänsch, B.; Martinez, P.; Uribe, D.; Zuluaga, J.; van Eldik, R.; Inorg. Chem. 1991, 30, 4555.

20. Bänsch, B.; Martinez, P.; Zuluaga, J.; Uribe, D.; van Eldik, R.; Z. Phys. Chem. (Munich) 1991, 170, 59.

21. Bänsch, B.; van Eldik, R.; Martinez, P.; Inorg. Chim. Acta 1992, 201, 75.

22. Martinez, P.; Zuluaga, J.; Uribe, D.; van Eldik, R.; Inorg. Chim. Acta 1987, 136, 11.

23. Martinez, P.; Zuluaga, J.; Kraft, J.; van Eldik, R.; Inorg. Chim. Acta 1988, 146, 9.

24. Xu, J.; Jordan, R. B.; Inorg. Chem. 1990, 29, 2933.

25. Tsukahara, K.; Yamamoto, Y.; Bull. Chem. Soc. Jpn. 1981, 54, 2642.

26. Martinez, P.; Zuluaga, J.; Uribe, D.; Z. Phys. Chem. Neue Folge 1983, 137, 43.

27. Dixon, D. A.; Sadler, N. P.; Dasgupta, T. P.; Trans. Met. Chem. 1995, 20, 295.

28. Kimura, M.; Yamamoto, M.; Yamabe, S.; J. Chem. Soc. Dalton Trans. 1982, 423.

29. Fornaro, A.; Tese de Doutorado, Instituto de Química da USP, São Paulo, SP. 1996.

30. Fornaro, A; Coichev, N.; J. Coord. Chem. 1998, no prelo.

31. Ghosh, S. K.; Gould, E. S.; Inorg. Chem. 1989, $28,1538$.

32. Pelizzetti, E.; Mentasti, E.; Pramauro E.; Inorg. Chem. 1976, 15, 2898.

33. Subba Rao, P. V.; Saradamba, G. V.; Ramakrishna, K.; Mohana Rao, K.; Subbaiah, K. V.; Indian J. Chem. 1989, 28A, 1060.

34. Salem, I. A.; Gemeay, A. H.; Trans. Met. Chem. 1996, 21,130 .

35. Mondal, B.; Kundu, S.; Banerjee, R.; Polyhedron 1997, 16, 2199.

36. Saha, B.; Gangopadhyay, S; Ali, M.; Banerjee, P.; J. Chem. Soc. Dalton Trans. 1995, 1083.

37. Iyun, J. F.; Ayoko, G. A.; Lawal, H. M.; Trans. Met. Chem. 1995, 20, 30.

38. Goddard, W. P.; Coupland, K.; Smith, J. A.; Long, R. G.; J. Nutr. 1997, 127, 177.

39. Olivares, M.; Pizarro, F.; Pineda, O.; Name, J. J.; Hertrampf, E.; Walter, T.; J. Nutr. 1997, 127, 1407. 
40. Hallberg, L.; Bibl. Nutr. Dieta 1995, 52, 20.

41. Hunt, J. R.; Gallagher, S. K.; Johnson, L. K.; Am. J. Clin. Nutr. 1994, 59, 1381.

42. Xu, J.; Jordan, R. B.; Inorg. Chem. 1990, 29, 4180.

43. Keypour, H.; Silver, J.; Wilson, M. T.; Hamed, M. Y.; Inorg. Chim. Acta 1986, 125, 97.

44. Laurence, G. S.; Ellis, K. J.; J. Chem. Soc. Dalton Trans. 1972, 1667.

45. Birus, M.; Kujundzic, N.; Pribanic, M.; Prog. React. Kinet. 1993, 18, 171 .

46. Beckford, F. A.; Dasgupta, T.P.; Stedman, G.; J. Chem. Soc. Dalton Trans. 1995, 2561.

47. Pelizzetti, E.; Mentasti, E.; Pramauro E.; Inorg. Chem. 1978, 17, 1181.

48. Hynes, M. J.; Kelly, D. F.; J. Chem. Soc., Chem. Commun. 1988, 849.

49. Grinstead, R. R.; J. Am. Chem. Soc. 1960, 82, 3464.

50. Ghosh, S. K.; Gould, E. S.; Inorg. Chem. 1989, $28,1948$.
51. Taqui Khan, M. M.; Martell, A. E.; J. Am. Chem. Soc. 1967, 89, 4176.

52. Taqui Khan, M. M.; Martell, A. E.; J. Am. Chem. Soc. 1967, 89, 7104.

53. Ogata, Y.; Kosugi, Y.; Tetrahedron 1969, 25, 4633.

54. Jameson, R. F.; Blackburn, N. J.; J. Inorg. Nucl. Chem. 1975, 37, 809.

55. Nord, H.; Acta Chem. Sce. 1955, 9, 442.

56. Sasso, M. G.; Quina, F. H.; Bechara, J. H.; Anal. Biochem. 1986, 156, 239.

57. Jameson, R. F.; Blackburn, N. J.; J. Chem. Soc. Dalton Trans. 1982, 9.

58. Shtamm, E. V.; Purmal, A. P.; Skurlatov, Y. I.; Int. J. Chem. Kinet. 1979, XI, 461.

59. Jameson, R. F.; Blackburn, N. J.; J. Chem. Soc. Dalton Trans. 1976, 534.

60. Harel, S.; J. Agric. Food Chem. 1994, 42, 2402.

61. Sarma, A. D.; Sreelakshmi, Y.; Sharma, R.; Phytochemistry 1997, 45, 671 . 